\title{
Tiefenregistrierende Nanohärtemessung - Verfahren, Beispiele und Anwendungsmöglichkeiten
}

\author{
Bodo Wolf, Ronald Ries, Marek Nowicki und Asta Richter
}

\section{Zusammenfassung}

Die tiefenregistrierende Nanohärtemessung hat sich in den letzten 10 Jahren zu einer Standardmethode der nanomechanischen Prüfung entwickelt. Einerseits erschließt sie Strukturgrößen von $10 \mathrm{~nm}$ bis $1 \mu \mathrm{m}$ Abmessung, andererseits liefert sie aber auch deutlich mehr Informationen als die klassische Härteprüfung. In der Arbeit wird die Methode der tiefenregistrierenden Härteprüfung aus der Sicht der technischen Realisierung, der Auswertung der Kraft-Tiefen-Kurven sowie der Interpretation der Ergebnisse dargestellt. Am Beispiel der Photoplastizität wird eine in situ-Modifizierung der mechanischen Eigenschaften vorgestellt, d. h. die Härte verändert sich reversibel durch Lichteinwirkung während der Messung. Ionenimplantation und UV-Exposition sind irreversible Methoden der Variation mechanischer Eigenschaften. Hier liefert der Vergleich von KraftTiefe-Kurven vor und nach der Behandlung wertvolle Informationen über das Materialverhalten.

\section{Einleitung}

Die klassische Härteprüfung ist ein Zwei-Schritt-Verfahren: Zunächst wird durch Eindrücken eines Prüfkörpers (Indenters) standardisierter Geometrie (Brinellhärte: Kugelabschnitt, Rockwellhärte: Kegel mit Kugelsegment an der Spitze, Vickershärte: vierseitige Pyramide) mit definierter Kraft in der Probe eine Vertiefung erzeugt. Im Schritt Zwei wird nach vollständiger Entlastung die erzeugte Delle mit einem Mikroskop aufgesucht und ausgemessen. Die so genannte Meyer-Härte ergibt sich aus dem Quotienten (aufgewandte Kraft/Projektionsfläche der Delle) [1]. Physikalisch gesehen handelt es sich um den mittleren Kontaktdruck, der im Kontaktbereich von Probe und Prüfkörper auftritt. Im Gleichgewichtsfall charakterisiert er das „Gegenhaltevermögen“ des Probenmaterials gegen die aufgebrachte Last.

Die Nachteile des traditionellen Prüfverfahrens sind eindeutig:

1) Es wird nur der Endzustand - also die Situation nach Aufbringen maximaler Kraft - dokumentiert. Die Entwicklung der Vertiefung kann nur durch eine Folge von Eindrücken steigender Kraft, aber an verschiedenen Orten, verfolgt werden.

2) Beim Übergang zu immer kleineren Kräften wird das Aufsuchen des Eindrucks immer muihsamer. Man muss vom Lichtmikroskop zu höherauflösenden Verfahren, z. B. zum Rasterelektronenmikroskop, übergehen. Bei rauhen Proben ist es unter Umständen völlig unmög- lich, die erzeugte Vertiefung von zufällig vorhandenen Unebenheiten zu unterscheiden.

3) Die Vorgehensweise ist diskontinuierlich, erfordert den permanenten Eingriff des Operators und eignet sich nicht für eine automatisierte Experimentfuihrung und Auswertung.

Abhilfe schafft die tiefenregistrierende Härtemessung. Hier werden während der Messung Kraft F und Tiefe $h$ simultan aufgezeichnet, und aus der erhaltenen KraftTiefe-Kurve $\mathrm{F}(\mathrm{h})$ können Härte und E-Modul gewonnen werden. Die F(h)-Kurve besteht mindestens aus zwei Segmenten: der Eindruckkurve, die eine Überlagerung von elastischer und plastischer Deformation ist, und der Entlastungskurve, die bei vielen Materialien rein elastisch, bei Polymeren zumeist viskoelastisch verläuft. Abb. 1 zeigt eine F(h)-Kurve für Quarzglas, also eines amorphen Materials. Der glatte Kurvenverlauf liefert hier vergleichsweise wenig Zusatzinformationen, das Glas verhält sich kontinuumsmechanisch. Viel interessanter sind $\mathrm{F}(\mathrm{h})$-Verläufe, die Diskontinuitäten aufweisen, z. B. Sprünge, Knicke, Buckel oder Schleifen. In Abb. 2 ist ein solcher Eindruckverlauf bei GaAs dargestellt, bei dem ein deutlicher Sprung in der Tiefe erfolgt. Derartige Unstetigkeiten lassen sich mit mikroskopischen Prozessen bei der Verformung korrelieren, z. B. mit der Bildung von Versetzungen [2], der Erzeugung von Rissen [3], dem Platzen des natürlichen Oxids beim Eindringen des Prüfkörpers, Grenzflächeneffekten beim Durchdringen von Schichtfolgen und anderen Erscheinungen. In Abb. 2 handelt es sich beispielsweise um Versetzungsnukleation, d. h. der Tiefensprung trennt rein elastische Verformung vor dem Sprung von Materialtransport uiber Versetzungsgleiten nach dem Sprung.

Die tiefenregistrierende Nanohärtemessung ist natürlich das Mittel der Wahl bei der Untersuchung von Strukturen mit nm-Abmessung. Wenn dünne Schichten charakterisiert werden sollen, darf man nicht mehr als etwa $10 \%$ der Schichtdicke eindrücken, um die Schichthärte zu erhalten. Noch größere Einschränkungen gibt es beim E-Modul, da die Ausdehnung des elastischen Feldes viel weiter reicht als die plastische Zone. Hier sollte die Eindrucktiefe $5 \%$ der Schichtdicke nicht uiberschreiten. Bei einem Gefuige aus kleinen Partikeln ist es erforderlich, dass die Eindruckabmessung klein gegenüber der Partikelgröße ist und auch hinreichend ,großer“ Abstand zur Grenze zum angrenzenden Partikel gewahrt wird [4]. Hier erzwingt also die Kleinheit der Struktur die Notwendigkeit winziger Härteeindrücke. Aber auch bei Untersuchung eines Einkristalls von $10 \mathrm{~cm}^{3}$ Volumen kann die Nanohärteprüfung Neues ans Licht bringen. 
Zwar kann auch die klassische Makrohärteprüfung bei einer solchen Probe sinnvolle Werte liefern, im Gegensatz zu den oben erwähnten Beispielen, aber in dem von der Makroprüfung erfassten Volumen werden sich immer viele Kristallbaufehler befinden. Letztlich sind es diese Baufehler, die für den Widerstand gegenuiber mechanischer Deformation, also für die Härte, ausschlaggebend sind. Bei der Nanohärteprüfung ist die Wahrscheinlichkeit hoch, dass das getestete Volumen keine oder fast keine Defekte enthält. Es müssen erst Defekte erzeugt werden, die dann wandern können und so den Materialtransport bewerkstelligen. Nanohärtewerte können somit um einen Faktor 10 oder noch höher liegen als Tabellenwerte, die durch herkömmliche Makroprüfung gewonnen wurden. Als Beispiel sei die Messung der Härte im stahlfarbenen Zentralteil der Ein-EuroMuinze zitiert, welches aus Cu75Ni25 besteht. In Tabelle 1 werden Härtewerte aufgelistet, die bei Eindrücken

\begin{tabular}{|l|l|l|l|l|l|l|l|l|}
\hline Eindrucktiefe $\mathrm{h}$ & $100 \mathrm{mn}$ & $8 \mathrm{mn}$ & $500 \mathrm{~nm}$ & $200 \mathrm{~nm}$ & $100 \mathrm{~nm}$ & $25 \mathrm{~nm}$ & $10 \mathrm{~nm}$ & $5 \mathrm{~nm}$ \\
\hline
\end{tabular}

\begin{tabular}{|l|l|l|l|l|l|l|l|l|}
\hline Härte $\mathrm{H} / \mathrm{GPa}$ & $0,98^{\star}$ & $1,9^{\star \star}$ & 3,2 & 4,0 & 5,1 & 6,2 & 7,2 & 9,5 \\
\hline
\end{tabular}

Tab. 1: Ermittelte Härtewerte im Zentralbereich der Ein-Euro-Münze (stahlfarbenes CU75Ni25-Gebiet) für verschiedene Eindrucktiefen (Quellen: *Bayerisches Münzamt; *** Vickers-Mikrohärte; alle anderen Daten aus BerkovichNanohärteprüfung)

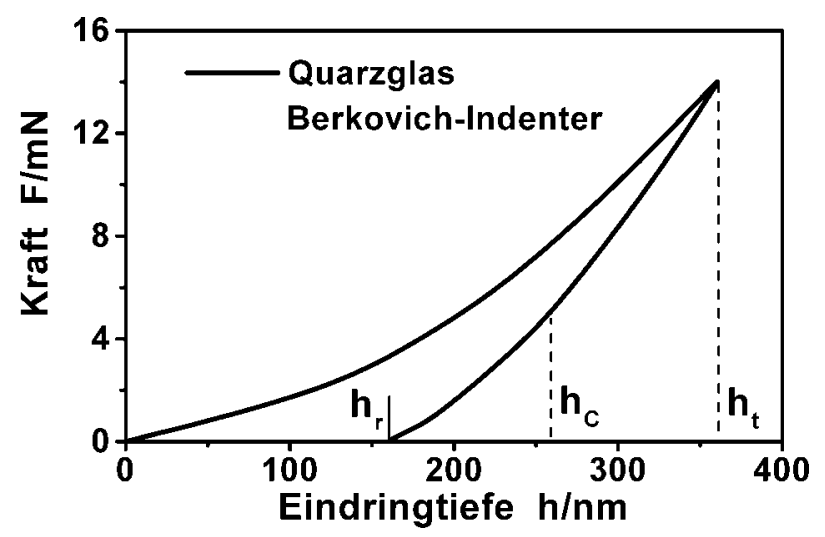

$A b b$. 1: Kraft $F$ als Funktion der Eindringtiefe $h$ für Quarzglas. Die Eindringkurve ist vergleichsweise glatt, typisch für amorphe Materialien. Der Indenter dringt maximal $361 \mathrm{~nm}$ ein $\left(h_{t}\right)$, nach Entlastung bleibt eine Vertiefung von $h_{r}=160 \mathrm{~nm}$ Tiefe zurück. Für die Bestimmung der Kontaktfläche ist die Kontakttiefe $h_{C}$ wesentlich, die etwa in der Mitte des Entlastungssegmentes angesiedelt ist $\left(h_{C} \approx\left(h_{r}+h_{t}\right) / 2\right)$.

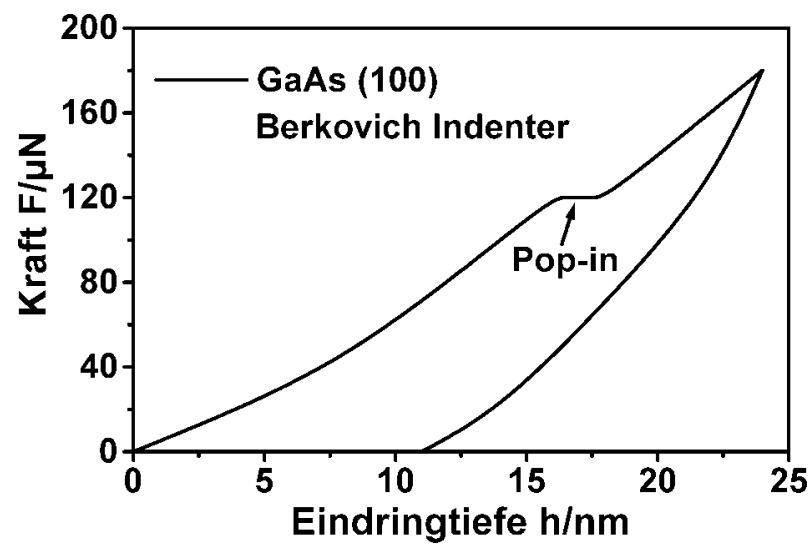

Abb. 2: Kraft als Funktion der Eindringtiefe für einkristallines GaAs (die Oberflächenorientierung ist (100)). Die Eindringkurve zeigt bei einer Tiefe von $16 \mathrm{~nm}$ einen Tiefensprung von $2 \mathrm{~nm}$ Amplitude. Dieser Sprung (,,popin“) markiert den Beginn plastischer Deformation durch Versetzungen. unterschiedlicher Tiefe in diesem Material gemessen wurden. Die Makrohärteprüfung liefert rund $1 \mathrm{GPa}$. Dieser Wert charakterisiert die Eigenschaften der Münze, „wenn sie unter den Hammer kommt“. Nanohärtemessungen liefern im Tiefenbereich von 100 bis $500 \mathrm{~nm}$ Härtewerte von 3 bis $6 \mathrm{GPa}$. Diese Daten charakterisieren die Oberflächeneigenschaften und sind somit aussagekräftig in Hinsicht auf das Abriebverhalten. Bei noch kleineren Tiefen (5 nm) steigt die Härte sogar auf 10 GPa an. Hier befindet man sich im Übergangsbereich von elastischer zu plastischer Deformation.

\section{Technische Realisierung der tiefen- registrierenden Nanohärteprüfung}

Es gibt zwei Kategorien von Nanohärteprüfern:

1) so genannte „stand-alone“-Geräte. Diese sind traditionelle Härteprüfer, die mit einer tiefenregistrierenden Messeinrichtung erweitert wurden.

2) Zusatzmodule zu einem Rastersondenmikroskop. Im Folgenden beschränken wir uns auf diese Geräteklasse, weil das Labor für Oberflächentechnik der Technischen Fachhochschule Wildau über ein derartiges Instrument verfuigt und weil es mit der Rastersondenabbildung einen weiteren (visuellen) Informationskanal zur Verfügung stellt.

Trägergerät der Wildauer Nanohärtemessung ist ein Rastersondenmikroskop „Nanoscope E“ der US-Firma Digital Instruments. An die Stelle des Biegebalkens zur Profilaufnahme ist nun ein elektrostatischer Dreiplattenkondensator (Transducer) getreten (Hersteller: HYSITRON Inc., Minneapolis, USA). Die Mittelplatte des Kondensators trägt den Eindruckdiamanten auf einem Stift (Abb. 3). Durch Anlegen einer Gleichspannung wird eine elektrostatische Kraft erzeugt, welche die Mittelplatte nach unten drüickt und den Eindruckkörper in die Probe presst. Gleichzeitig ändert sich die Kapazität des Kondensators infolge der Mittelplattenverschiebung. Primäre Messwerte sind also Spannung U und Kapazitätsänderung $\Delta \mathrm{C}$. Diese werden in Kraft $\mathrm{F} \sim \mathrm{U}^{2}$ und Tiefe $\mathrm{h} \sim \Delta \mathrm{C}$ umgerechnet. Maximal können $600 \mathrm{~V}$ Gleichspannung angelegt werden (bei höheren Werten besteht die Gefahr des elektrischen Durchschlags, die Feldstärke erreicht bei $600 \mathrm{~V}$ einen Wert von 1,5 Millionen Volt pro Meter). Daraus ergibt sich mit geometrischen Kondensatordaten (Plattenfläche $0,7 \mathrm{~cm}^{2}$, Plattenabstand $0,12 \mathrm{~mm}$ ) eine Maximalkraft von $6 \mathrm{mN}$, also die Gewichtskraft von 0,6 g. Traditionelle Makrohärteprüfer arbeiten mit Kräften, denen Massen von einigen $\mathrm{kg}$ bis zu einer Tonne entsprechen. Die übliche Mikrohärteprüfung reicht zwar bis zu einer unteren Lastgrenze von $5 \mathrm{mN}$ (also bis zu unserer oberen Lastgrenze) herab, allerdings muss man bei der Wahl der Materialien hier weitere Einschränkungen hinnehmen. Eine Eindruckkraft von $5 \mathrm{mN}$ - aufgebracht auf ein Polymer - vermag einen Eindruck von einigen $\mu \mathrm{m}$ Abmessung zu erzeugen, der lichtoptisch gerade noch erfassbar und auswertbar ist, während die gleiche Kraft bei harten Materialien, wie z. B. Titannitrid, Saphir oder DLC, keinen den Mitteln der Mikrohärteprüfung zugänglichen Eindruck hinterlässt. 
Eine umfassende Beschreibung des Transducers gibt Literaturstelle [5].

Aus der Eindrucktiefe h kann auf die Eindruckfläche A(h) und somit auf die Härte $\mathrm{H}=\mathrm{F} / \mathrm{A}$ geschlossen werden, wenn man die Form des Eindruckkörpers kennt. Eine interne Indenterkalibrierung nutzt die Methode von Oliver und Pharr [6] zur Bestimmung der Indenterform. Dabei werden Eindrücke unterschiedlicher Kraft und Tiefe in ein Standardmaterial (z. B. Quarzglas) gesetzt und die Flächenfunktion $\mathrm{A}(\mathrm{h})$ so bestimmt, dass die richtigen Werte von E-Modul E und Härte $\mathrm{H}$ für das Standardmaterial erhalten werden.

Neben der F(h)-Kurve ist das Abbilden des Eindrucks der zweite wichtige Informationskanal. Dabei wird die Eindruckspitze selbst als Abtastnadel benutzt. Die Bilderzeugung kann wie beim Rasterkraftmikroskop (AFM) in zwei Modi erfolgen. Beim Modus konstanter Kraft wird der gesamte Plattenkondensator horizontal zeilenförmig so über die Probe gefahren, dass die Mittelplatte in ihrer vertikalen Position verbleibt, der Diamant mit konstanter geringer Auflagekraft über das Probenprofil gleitet. Das Auf- und Abbewegen des gesamten Transducers, das mit einem Piezosteller bewerkstelligt wird, ist zugleich ein Abbild der Probenoberfläche. Beim Modus konstanter Höhe wird der Kondensator horizontal in konstanter Höhe verfahren (der vertikale Piezosteller ist inaktiv), und das Probenprofil schiebt die aufliegende Abtastnadel samt der Kondensatormittelplatte innerhalb des Kondensators nach oben und unten. Dabei ändert sich permanent die Kapazität, und aus dem Kapazitätsverlauf kann die Oberflächengeometrie rekonstruiert werden. Zur Abbildung verwenden wir primär die Methode konstanter Kraft; den Modus konstanter Höhe setzen wir für Kalibrierzwecke ein.

Die Möglichkeit einer in situ-Abbildung ist in zweierlei Hinsicht gewinnbringend: Erstens ermöglicht sie eine Feinpositionierung des Indenters an der gewünschten Stelle, zweitens kann die Eindruckvertiefung visualisiert

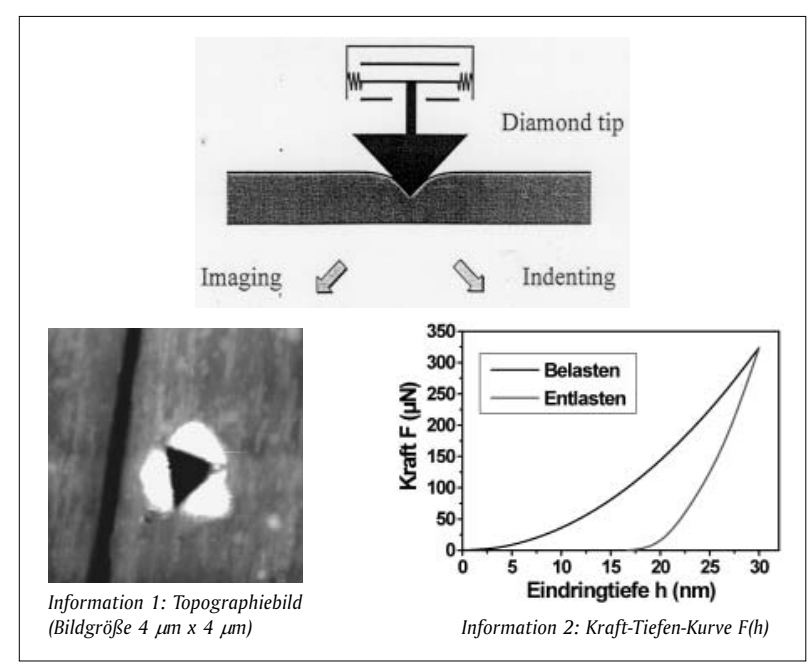

Abb. 3: Experimenteller Aufbau der tiefenregistrierenden Nanohärteprüfung: Ein Dreiplattenkondensator trägt auf der Mittelplatte einen Stift, auf dem sich der Eindruckprüfkörper (,Diamond tip“) befindet. Als Informationskanäle erhält man ein Bild der Eindruckvertiefung (links), sowie die Kraft Tiefe - Kurve (rechts). werden. Bei duktilen Substanzen kann man häufig Wallbildungen (Herausquetschen von Material) im Umfeld der Eindruckdelle erkennen (Abb. 3), spröde Proben zeigen Risse oder Ausbrüche. Daraus können auch weitere quantitative Parameter, wie z. B. die Bruchzähigkeit, abgeleitet werden.

\section{Härteeindruckkörper}

Selbstverständlich muss der Prüfkörper härter als das zu testende Material sein. Im Allgemeinen verwendet man Diamant, da dieser die höchste Härte der bekannten und verfügbaren Materialien hat. Bei der Nanohärteprüfung braucht der Prüfdiamant auch nur sehr klein zu sein (weniger als $0,1 \mathrm{~mm}^{3}$ ). Leider erweist sich die Härte als von der Geometrie des Prüfkörpers (Indenters) abhängig. Deshalb wurden Standard-Geometrien eingefuihrt. Besonders günstig sind Pyramiden und Kegel, da sie geometrisch selbstähnlich sind [7]: Das Verhältnis Höhe $\mathrm{h} /$ Breite b ist bei ein und demselben Eindruckkörper höhenunabhängig. Wir finden

$\cot \alpha=2 \mathrm{~h} / \mathrm{b}$

d. h. die Geometrie wird nur durch den halben Öffnungswinkel $\alpha$ beschrieben. Diese Selbstähnlichkeit wird z. B. bei Kugeleindrücken verletzt. Es seien $\rho$ der Radius des erzeugten Eindrucks, R der Radius der Kugel (bzw. des Kugelsegmentes an einem Kegel- oder Pyramidenstumpf), und h die Eindrucktiefe. Bei einem flachen Eindruck $(\mathrm{h}<<\mathrm{R})$ gilt:

$\rho^{2}=2 \mathrm{Rh}$

$\cot \alpha=\rho / h=(2 R / h)^{1 / 2}$

Der effektive Öffnungswinkel sinkt mit zunehmender Tiefe h, die Delle wird spitzer. Aus der Plastizitätstheorie des Kontinuums ist bekannt, dass der Verformungsgrad und damit die Härte mit größer werdendem $\alpha$ abnehmen, sodass man bei Kugeleindrücken eine Härtezunahme mit der Tiefe verzeichnet (Meyer-Verhalten) [7]. Dagegen liefern Eindruckkörper mit gleichem $\alpha$ tiefenunabhängige Makrohärtewerte. Von diesem makroskopischen kontinuumsmechanischen Gesetz findet man deutliche Abweichungen bei der Nanohärte, die in der atomaren, diskreten Struktur des Messobjektes begründet sind. Insbesondere spielt die Häufigkeit von Defekten im Indentierungsbereich eine große Rolle, wie schon in der Einleitung beschrieben. Für die Nanohärtemessung haben sich dreiseitige Pyramiden als bevorzugte Eindruckkörper durchgesetzt. Sie liefern beim Eindrücken ein gleichseitiges Dreieck als Vertiefungsfigur (siehe Abb. 3). Unterschiedlich sind die Öffnungswinkel. Die vollen Öffnungswinkel $2 \alpha$ betragen beim BerkovichIndenter $2 \alpha=136^{\circ}$, beim Würfelspitzenindenter $2 \alpha=$ $90^{\circ}$ und beim $60^{\circ}$-Indenter $2 \alpha=60^{\circ}$. Je kleiner $\alpha$, desto größer die Rissbildung und Zersplitterung bei spröden Materialien. Deshalb bevorzugt man den BerkovichIndenter, da dieser stumpf verformt statt spitz zerschneidet. Andererseits ist die Abbildungsqualität bei kleinem $\alpha$ deutlich besser. Ein Kompromiss ist der Würfelspitzen- 
indenter, $d$. h. eine Pyramide, die beim symmetrischen Abschneiden einer Würfelecke entsteht. Kegel sind wegen der Rotationssymmetrie optimal, lassen sich aber viel schwieriger in der erforderlichen Qualität aus Diamant schleifen.

Neben der Idealgeometrie spielt der Krümmungsradius der Spitze eine große Rolle. Er entscheidet darüber, bei welcher Eindrucktiefe h der Übergang von rein elastischer zu plastischer Deformation erfolgt. Bei Einkristallen findet der in Abb. 2 gezeigte Tiefensprung (,pop-in“) bei Tiefen von 10 bis $15 \%$ des Indenterradius R statt. Ist $\mathrm{R}$ also groß, so muss man tief eindrüicken, bevor die plastische Deformation beginnt. Dann ist das Verformungsvolumen zu groß, um defektarm zu sein - eine Diskontinuität, ein pop-in, wird nicht beobachtet. Die rein elastische geht kontinuierlich in eine plastische Deformation über. Bei kleinem R setzt die plastische Deformation bei viel kleinerer Tiefe, $d$. h. bei einer größeren Wahrscheinlichkeit von Defektarmut im verformten Bereich ein. Aus Gleichung (2) folgt

$\mathrm{A}(\mathrm{h})=\pi \rho^{2}=2 \pi \mathrm{Rh}$

Der Krümmungsradius R ergibt sich aus der Flächenfunktion $\mathrm{A}(\mathrm{h})$ gemäß

$\mathrm{R}=\mathrm{A}(\mathrm{h}) / 2 \pi \mathrm{h}$

bei kleinen Werten $\mathrm{h}<<\mathrm{R}$. Bei dem von uns verwendeten Berkovich-Eindruckkörper beträgt $R=115 \mathrm{~nm}$.

\section{Anisotropie mechanischer Eigenschaften}

Bedingt durch den Gitteraufbau kristalliner Festkörper sind viele Materialeigenschaften richtungsabhängig (anisotrop). Die meisten Werkstoffe haben jedoch ein polykristallines Gefuige, d. h. sie bestehen aus kleinen Körnern, die man als Einkristalle betrachten kann. Makroskopische Tests, wie der Zugversuch an einer Stabprobe, mitteln nun über die Körner in einem vom Test erfassten Gebiet. Wenn die Orientierung der Körner völlig wahllos ist, dann liegt Quasiisotropie vor. Mitunter gibt es aber auch eine Bevorzugung bestimmter Richtungen (Textur). Mechanische Bearbeitung mit ausgeprägter Vorzugsrichtung, z. B. Walzen von Blechen oder Ziehen von Drähten, kann zu einer solchen Textur fuihren.

Mit Ausnahme extrem nanokristalliner Materialien wird die Nanohärtemessung an kristallinen Substanzen jeweils individuelle Körner treffen, d. h. es werden Einkristalle unterschiedlicher Orientierung getestet. Insofern muss man bei der Nanohärteprüfung polykristallinen Materials mit einer großen Streuung der Messdaten rechnen, wenn die Messpunkte über einen größeren Oberflächenbereich verteilt sind (mesoskopische Messpunktverteilung). Identische Ergebnisse sind nur bei Verteilung der Messorte innerhalb desselben Korns zu erwarten. Das Einsatzpotenzial der Nanohärteprüfung liegt deshalb auf den Gebieten der Einkristalle sowie nanokristalliner und glasartiger/amorpher Materialien.
Bei grobkörnigen Polykristallen sollte daher die Makrohärteprüfung wegen der ihr inhärenten Mittelungseigenschaft uiber viele Körner bevorzugt werden.

Bei Verwendung von Eindruckkörpern, die nicht rotationssymmetrisch sind, wie Eindruckpyramiden, wird die gemessene Härte auch von der azimuthalen Orientierung zwischen Probe und Indenter abhängen. Dies trifft nicht auf den E-Modul zu. Wenn man also die Probe um ihre Oberflächennormale dreht, so wird man eine periodische Änderung der Härte mit dem Drehwinkel feststellen [8]. Die Periodenlänge hängt von der Symmetrie von Probe und Indenter ab. Testet man mit einem Berkovich-Indenter (3-zählige Symmetrie) eine (111)-Fläche (ebenfalls 3-zählig), dann wird die Periode $120^{\circ}$ betragen. Bei der Prüfung einer (100)-Fläche (4-zählig) liegt eine Symmetrieuiberlagerung vor. Die Fehlanpassung beträgt $120^{\circ}-90^{\circ}=30^{\circ}$, sodass man eine Pseudozwölfzähligkeit der Härte erwarten kann. Vorstehende Betrachtung hat folgende Konsequenz: Will man mit einem nichtrotationssymmetrischen Eindruckkörper einen Härtevergleich von Oberflächen verschiedener Orientierung vornehmen, so muss man Messreihen mit schrittweiser Verdrehung der Probe um ihre Achse aufnehmen und kann nur diese vergleichen. Die genannte Schwierigkeit entfällt, wenn man einen Kegel bzw. eine Kugel als Eindruckkörper verwendet. Hier können Eindrücke in Oberflächen unterschiedlicher Orientierung unmittelbar hinsichtlich der Härte verglichen werden. Die Anisotropie erweist sich also als ein Erschwernis bei der nanomechanischen Charakterisierung polykristalliner technischer Werkstoffe, da eine umfangreiche Statistik erforderlich wird, um aussagekräftige Mittelwerte zu erhalten. Andererseits ist dies natürlich auch eine Informationsquelle für den Physiker/Werkstoffwissenschaftler, welchen die atomistische Struktur des Materials interessiert. Zur Untersuchung mechanischer Anisotropie wurde speziell der Knoop-Indenter entwickelt. Dabei handelt es sich um eine vierseitige Pyramide mit rautenförmigem Querschnitt (Diagonalenverhältnis 1:7). Durch seine zweizählige Symmetrie stört er die Symmetrie der Messprobe besonders wenig.

Experimentell wurden Eisen-Einkristalle der Orientierung (100), (110) und (111) getestet. Eisen hat einen elastischen Anisotropiefaktor A = 2.5 [9], d. h. die E-Moduli unterschiedlich orientierter Kristalle bei einachsiger Belastung sind sehr verschieden. Da jedoch bei der Indentierung ein komplizierter mehrachsiger Belastungsfall vorliegt, werden die elastischen Eigenschaften über alle Richtungen gewichtet gemittelt. Das Gewicht der Eindruckrichtung ist dabei nur geringfuigig höher als das aller anderen Richtungen, sodass die Richtungsvariation der gemessenen elastischen Moduli wesentlich geringer ausfällt als die Variation der E-Moduli bei einachsiger Belastung [10]. Den aus dem Nanohärteexperiment bestimmten elastischen Modul nennt man Indentierungsmodul $\mathrm{E}_{\text {ind }}$. Für eine isotrope Probe ist er mit dem herkömmlichen E-Modul identisch. Für einen Einkristall erhält man einen Indentierungsmodul, der nahe am Hillschen Mittel liegt, also dem E-Modul einer zugeordneten isotropen polykristallinen Probe ent- 
spricht. Die Theorie von Vlassak und Nix [10] sagt voraus, dass die Indentierungsmoduli bei Eisen von (100) über (110) zu (111) anwachsen, wobei zwischen (100) und (111) nur $12 \%$ Unterschied zu erwarten sind. Dies wurde auch im Experiment bestätigt (Abb. 4). Zum Einsatz kam ein Berkovich-Indenter. In Abb. 4 wurde der gemessene Indentierungsmodul als Funktion der Eindrucktiefe aufgetragen. Da es sich um eine tiefenhomogene Probe handelt, sollten die Werte konstant sein. Der Anstieg ist durch einen Messartefakt begründet. Mit zunehmender Eindrucktiefe kommt es zu einer verstärkten Bildung von Materialaufwürfen um die Eindruckvertiefung. Dadurch wird die Kontaktfläche erhöht, was in der Auswertung nach Oliver und Pharr nicht berücksichtigt wird. Für den Indentierungsmodul $E_{\text {ind }}$ liegt eine Proportionalität

$\mathrm{E}_{\text {ind }} \sim 1 / \mathrm{A}^{1 / 2}$

bezüglich der Kontaktfläche A vor, sodass zu kleine Kontaktflächen den Indentierungsmodul scheinbar ansteigen lassen. Da sich die Wallbildung bei den einzelnen Oberflächen vermutlich in gleicher Weise entwickelt, wirkt dieser Artefakt als systematischer Fehler, sodass ein Unterschied von $12 \%$ in den Indentierungsmoduli über verschiedene Tiefen erhalten bleibt.

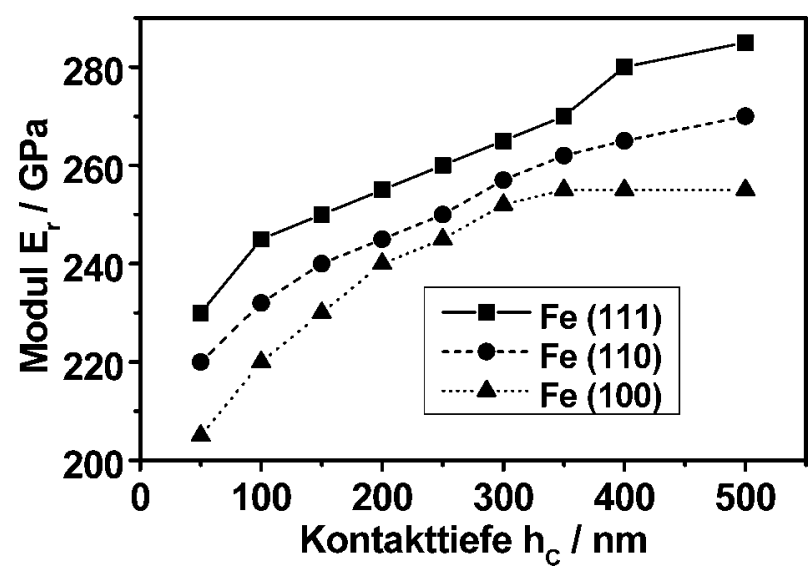

Abb. 4: Indentierungsmodul von einkristallinem Eisen (Oberflächenorientierung (100), (110) und (111) ) aus Nanoindentierungsmessungen mit einem Berkovich- und einem Konus-Indenter (Mittelwerte aus mehreren Messungen). Die scheinbare Zunahme von $E_{r}$ mit der Tiefe ist ein Artefakt, der durch zunehmende Wallbildung hervorgerufen wird.

\section{Reversible Änderung nanomechanischer Eigenschaften: Photoplastizität}

Photoplastizität ist ein Beispiel für eine reversible in situBeeinflussung der Härte während der Messung. Unter Beleuchtung ändert sich die Härte, und der Effekt verschwindet bei Abschalten der Lichtquelle: Eine irreversible Materialänderung findet nicht statt. Die in diesem Abschnitt vorgestellten Untersuchungen wurden an der TU Dresden, Institut für Kristallographie und Festkörperphysik (IKFP), durchgefuihrt. Dabei wurde ein zum Wildauer Nanohärteprüfer äquivalentes Gerät verwendet, das aber durch eine offene Bauweise die Messung größerer Proben bzw. Manipulationen während der Messung ermöglicht.

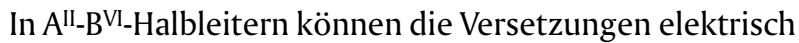
geladen sein. Dies hängt mit dem polaren Charakter der (111)-Ebenen als Gleitebenen zusammen, die entweder nur aus $\mathrm{A}^{\mathrm{II}}$ - oder nur aus $\mathrm{B}^{\mathrm{VI}}$-Atomen bestehen. Wenn die Elektronegativitätsdifferenz zwischen A und B hinreichend groß ist, dann liegt trotz kovalenter Bindung ein teilweiser Ladungstransfer zwischen A und B vor, mithin tragen die A- und B-Ebenen partielle Ladungen. Durch Einstrahlung von Licht kann man nun den internen lichtelektrischen Effekt initiieren: Man hebt Ladungsträger (Elektronen bzw. Löcher) ins Leitungsband an, die nun zu den Versetzungen wandern und diese umladen können. Damit ändern sich die elektrostatischen Kräfte zwischen den Versetzungen selbst, zwischen Versetzungen und dem Gitter oder zwischen geladenen Versetzungen und elektrisch aktiven Punktdefekten $[11,12]$. Im Ergebnis dessen erhält man eine modifizierte Fließspannung und somit eine Variation der Härte. Bei II-VIIHalbleitern liegt zumeist ein positiver photoplastischer Effekt (PPE) vor, d. h. die Härte steigt bei Beleuchtung an. Abgesehen von Eiern, die bei Erwärmung hart werden, zeigen die meisten Materialien eine Erweichung bei Erwärmung, sodass man die Härtesteigerung beim positiven PPE relativ leicht von thermischen Effekten trennen kann. In Abb. 5 sind zwei Eindruckkurven in $\mathrm{ZnSe}$ gezeigt. Bei gleicher Maximalkraft dringt der Indenter bei Dunkelheit tiefer in die Probe ein als im Zustand der Beleuchtung mit Laserlicht. Die Härtesteigerung lässt sich aus der Kurvenshift in folgender Weise abschätzen: Bei einer Pyramide werden Querschnittsfläche A und Tiefe h durch eine Beziehung

$\mathrm{A} \sim \mathrm{h}^{2}$

verknüpft. Für die Härte $H$ folgt

$\mathrm{H} \sim \mathrm{F} / \mathrm{h}^{2}, \Delta \mathrm{H} / \mathrm{H}=\Delta \mathrm{F} / \mathrm{F}-2 \Delta \mathrm{h} / \mathrm{h}$

Eine Reduktion der Eindringtiefe um $10 \%$, wie in Abbildung 5 gezeigt, hat folglich rund $20 \%$ Härtesteigerung zur Folge.

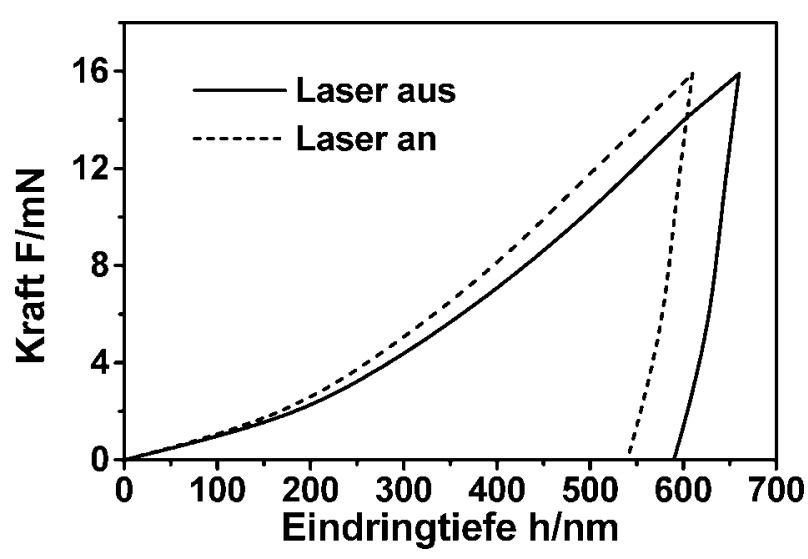

Abb. 5: Vergleich der Eindringtiefen bei Nanohärteprüfung von ZnSe in Dunkelheit (,Laser aus“) und bei Bestrahlung mit grünem Licht $(\lambda=543$ $\mathrm{nm}$ ). In der Dunkelheit ist die Eindringtiefe etwa 10 \% erhöht, die Härte um $20 \%$ erniedrigt.

Wird die Lichtquelle abgeschaltet, dann stellt sich der alte Ladungszustand der Versetzungen und damit auch die alte Härte wieder ein. Dies geschieht nicht augen- 
blicklich, sondern erfordert eine gewisse Relaxationszeit. Daher existiert der photoplastische Effekt eine Zeit lang mit verminderter Stärke weiter (photoplastische Nachwirkung) [12].

\section{Irreversible Änderung nanomechanischer Eigenschaften}

\subsection{Ionenimplantation}

Ionenimplantation führt zu massiven Strukturänderungen. In Polymeren resultiert die Ionenimplantation in einer räumlichen Vernetzung der Polymerketten (cross-linking), verbunden mit einer Steigerung von Härte und E-Modul [13]. Außerdem wird das viskose Verhalten reduziert. Viskoses Verhalten manifestiert sich im Nanohärtetest durch eine Hystereseschleife beim Entlasten und Wiederbelasten. Die Entlastungskurve ist nicht rein elastisch, sondern hat anelastische Anteile. Folglich fallen Entlastungs- und Wiederbelastungskurve nicht zusammen. Der Flächeninhalt der sich bildenden Schleife ist dann ein Irreversibilitätsmaß, also Ausdruck z. B. der Viskosität. Abb. 6 zeigt einen Multi-Eindruck

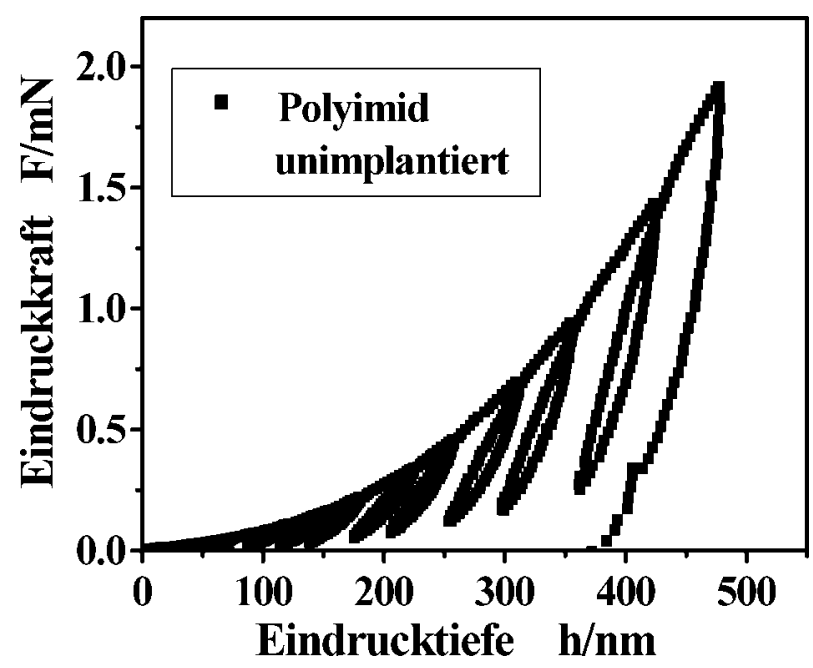

Abb. 6: Multi-Eindruck in unbehandeltem Polyimid. Die Viskosität des Polymers führt zu Hystereseschleifen bei Entlastung und Wiederbelastung.

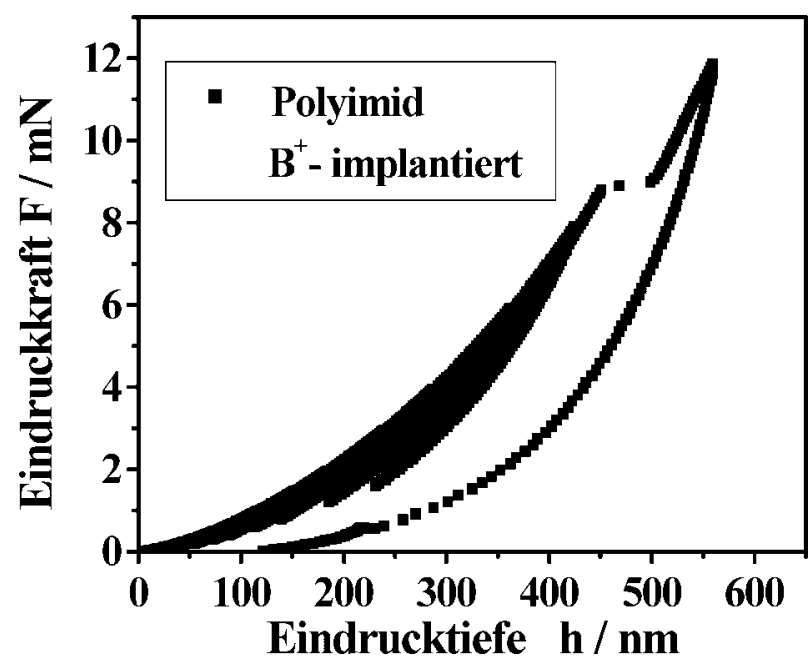

Abb. 7: Multi-Eindruck in ein Polyimid nach Ionenbeschuss (Energie $180 \mathrm{keV}$, Dosis $10^{16} \mathrm{~cm}^{-2}$ ). Die ionenmodifizierte Schicht verhält sich stärker elastisch, Hystereseschleifen sind verschwunden.

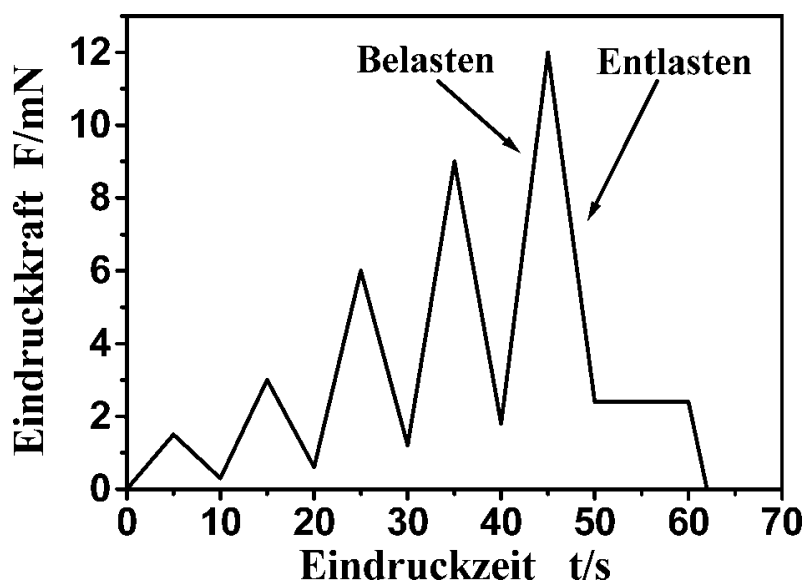

Abb. 8: Kraft-Zeit-Belastungsschema für einen Multi-Eindruck nach Abb. 7 u. 9

in ein unbehandeltes Polymer, Abb. 7 wurde nach Ionenimplantation aufgenommen. Das zugehörige Kraft-ZeitRegime bei der Eindruckprüung ist in Abb. 8 gezeigt. Man resümiert folgende Effekte:

- Reduktion der Eindringtiefe bei gleicher Kraft $\rightarrow$ Härtesteigerung (die Eindrucktiefe bei $2 \mathrm{mN}$ Last reduziert sich um die Hälfte beim Vergleich der Bilder 6 und 7)

- Zunahme des E-Moduls

- Verschwinden der Hystereseschleifen

Hystereseschleifen können auch Ausdruck von Phasenumwandlungen beim Entlasten und Wiederbelasten sein. Die Diamant- bzw. Zinkblendestruktur, in der viele Halbleiter kristallisieren, ist relativ anfällig auf Phasenumwandlung [14]. Wenn die kritische Schubspannung zur Bewegung von Versetzungen viel kleiner als der erforderliche Initiierungsdruck zur Phasenumwandlung ist, dann basiert die plastische Verformung auf Versetzungsbewegung allein, und Phasenumwandlungen treten beim Indentieren nicht auf. Dies trifft z. B. auf GaAs oder InP zu. Hier kann man die Phasenumwandlung nur durch hydrostatische Kompression aus allen Richtungen erreichen, bei der ein „Ausweichen“ der Atome verhindert wird. Liegen kritische Schubspannung und Phasenumwandlungsdruck bei ähnlichen Werten, dann kann die Phasenumwandlung zur plastischen Verformung beitragen, wie man dies bei Si und InSb beobachtet. Die Phasenum- bzw. Ruickwandlung manifestiert sich in einer deutlichen Hystereseschleife, wie aus Abb. 9a für InSb ersichtlich. Nach Ionenimplantation verschwindet die Hystereseschleife vollständig (Abb. 9b). Dieses Ergebnis kann man als Folge einer Amorphisierung der Probe durch die Implantation deuten.

\subsection{Bestrahlung mit UV-Licht}

Polymere haben viele traditionelle Werkstoffe verdrängt und gehören heute zu den häufigsten im Alltag zu finden Materialien. UV-Strahlung ist ein Faktor, der im Zusammenhang mit Polymeren eine besondere Rolle spielt: Einerseits ist UV im Sonnenlicht enthalten und zählt für Polymere im „Außeneinsatz“ zu den wichtigsten destruktiven Umweltfaktoren [15]. Andererseits wird UV auch bewusst in der Polymerverarbeitung (z. B. Aushärten von Lacken) eingesetzt, bzw. im Polymerdesign (verrottbare Polymere als eine Teillösung des Entsorgungsproblems) berücksichtigt. 


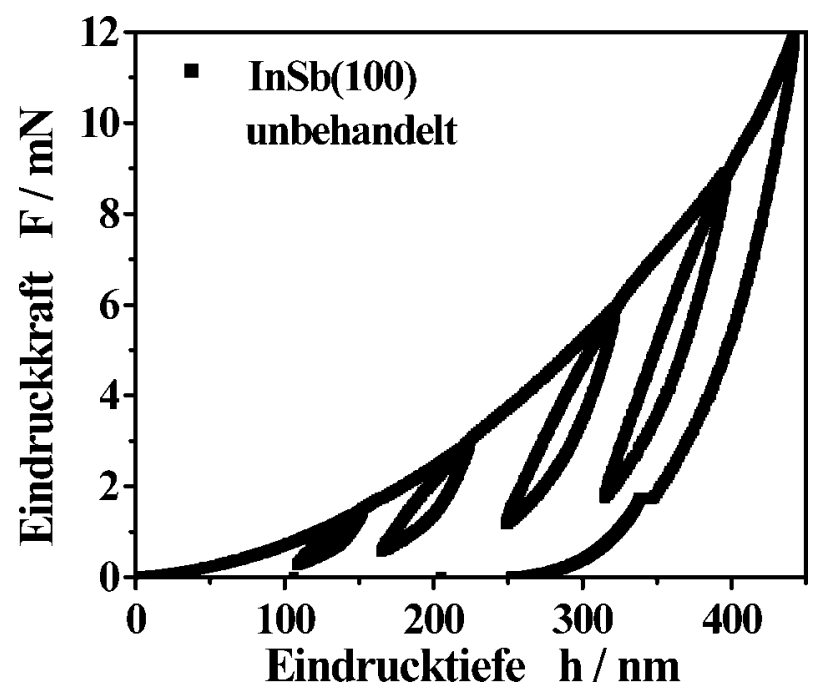

Abb. 9a: Multi-Eindruck in einkristallinem InSb mit deutlicher Ausprägung von Hystereseschleifen bei Entlastung und Wiederbelastung, hervorgerufen durch druckinduzierte Phasenumwandlung.

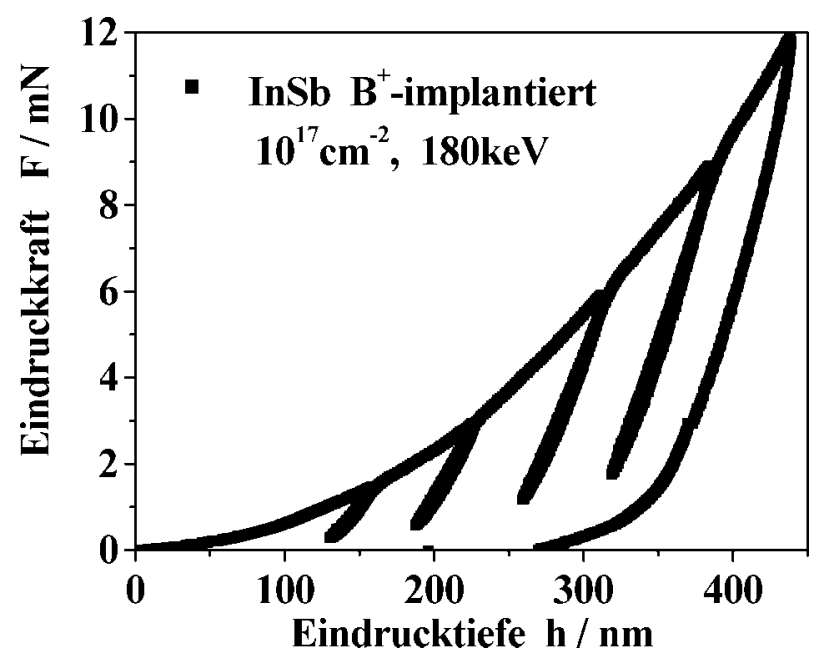

Abb. 9b: Multi-Eindruck in InSb nach Ionenimplantation. Durch partielle Amorphisation wurde die druckinduzierte Phasenumwandlung unterdrückt, Hystereseschleifen treten nicht auf.

In einer gemeinsamen Studie der TFH Wildau und der TU Poznan wurde der Einfluss von UV auf die nanomechanischen Eigenschaften einer Reihe verschiedener Polymere untersucht. Im Folgenden wird über Polyethylenoxid (PEO) und Polyvinylchlorid (PVC) berichtet. Die Proben wurden ohne UV-Exposition sowie nach polychromatischer Bestrahlung mit einer Hg-Niederdruckentladungslampe mit verschiedenen Expositionszeiten untersucht. $\mathrm{Hg}$ hat im nahen UV eine Reihe von Spektrallinien, insbesondere bei $\lambda=254 \mathrm{~nm}$. Die Bestrahlungsdichte betrug dabei $4,5 \mathrm{~mW} / \mathrm{cm}^{2}$.

PEO ist ein teilkristallines Polymer, das einen Anteil von 70 bis $90 \%$ kristallines Gefuige im Volumen aufweisen kann [16]. Durch UV-Bestrahlung wird in PEO der kristalline Anteil erhöht. Dies zeigte sich auch lichtmikroskopisch in der Oberflächenmorphologie: dentritisches Wachstum von Kristalliten mit zunehmender Bestrahlungsdauer. Wie aus Bild 10a (Gegenüberstellung der F(h)-Kurven vor und nach Bestrahlung) hervorgeht, resultiert die UV-Exposition in einer reduzierten Eindringtiefe (erhöhten Härte) sowie einer steileren Ent- lastungskurve (erhöhtem E-Modul). Bei einem Multi-Eindruck bilden sich Entlastungs-Wiederbelastungs-Hystereseschleifen, die das viskose Verhalten des Polymers zum Ausdruck bringen. Die Fläche der Hystereseschleife ist die Verlustenergie, die bei Entlastung und Wiederbelastung irreversibel in Wärme umgesetzt wird. Diese Verlustenergie ist für die Erwärmung bei zyklischer Beanspruchung, für Kriechen und Ermüdungsversagen bedeutsam. Quantitativ lässt sich der inelastische Energieverlust durch das Verhältnis $\mathrm{W}_{\text {loss }} \mathrm{W}_{\text {elast }}$ sowie durch den Verlustwinkel

$\tan \gamma_{\text {loss }}=\mathrm{W}_{\text {loss }} / \mathrm{W}_{\text {elast }}$

beschreiben. Dabei sind $\mathrm{W}_{\text {loss }}$ die Verlustarbeit und $\mathrm{W}_{\text {elast }}$ die elastisch gespeicherte Energie. Gleichung 9 repräsentiert dann ein Dreieck in der komplexen Ebene, wobei $W_{\text {elast }}$ als Realteil und $W_{\text {loss }}$ als Imaginärteil einer komplexen (viskos-inelastischen) Verformungsarbeit aufgefasst werden. Die UV-Bestrahlung resultierte auch in einer Verringerung von $\mathrm{W}_{\text {loss }}$, also in einem verminderten viskosen Verformungsverhalten. Die Abnahme der Hysteresefläche kann visuell in Abb. 10b erfasst werden, wo $\mathrm{F}(\mathrm{h})$-Kurven eines Multiindents vor und nach Bestrahlung in einem Diagramm vereinigt sind.

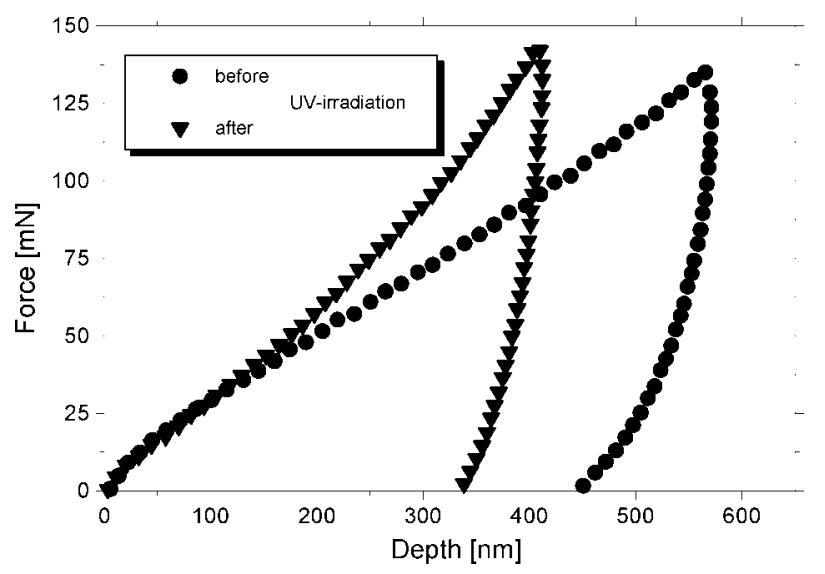

Abb. 10a: Vergleich der Eindruckkurven in PEO vor und nach Bestrahlung (10h Expositionsdauer). Die reduzierte Eindrucktiefe nach Bestrahlung weist auf erhöhte Härte hin, während der steilere Abfall der Entlastungskurve ein Maß für angestiegene Steifheit (vergrößerter E-Modul) ist.

Im Gegensatz zu PEO ist PVC ein vollständig amorphes Polymer $[17,18]$. Auch hier resultierte die UV-Exposition in einem Härteanstieg sowie einer reduzierten Viskosität. Der E-Modul blieb dagegen von der Bestrahlung weitgehend unbeeinflusst. Die Abbildung 11 zeigt Härte und E-Modul als Funktion der Expositionsdauer für PEO und PVC. Man erkennt, dass noch keine Sättigung des Härtungseffektes bei den verwendeten Dosen eingetreten ist. Die Härtung bei PVC wird durch eine räumliche Vernetzung der Polymerketten (cross-linking) erreicht [19].

Der Unterschied im Verhalten des E-Moduls kann wie folgt interpretiert werden. Der E-Modul ist ein Druck (der erforderliche Druck, um eine Stabprobe elastisch auf die Hälfte zu verkürzen, wenn dies möglich wäre) 


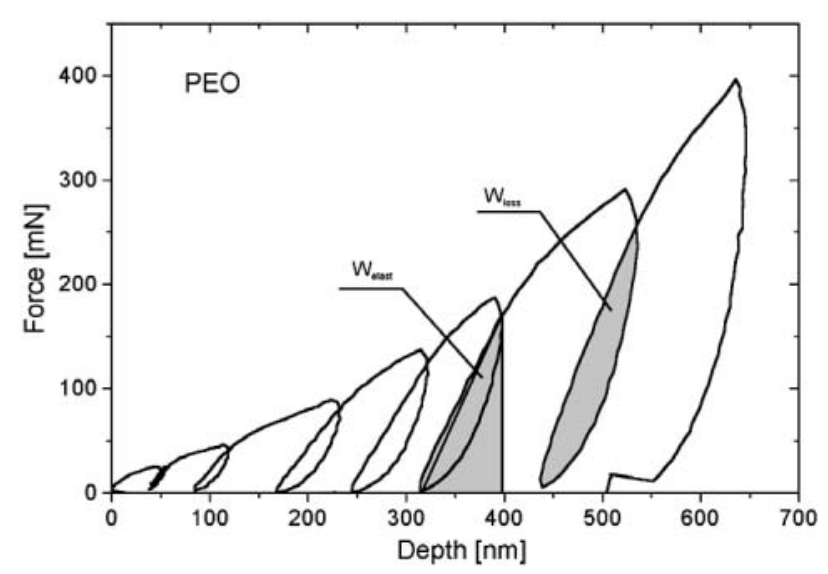

Abb. 10b: Multiindent in PEO mit Ausprägung von Hystereseschleifen. $W_{\text {loss }}$ repräsentiert die während eines Zyklus von Entlastung und Wiederbelastung irreversibel umgewandelte mechanische Energie. $W_{\text {elast }}$ stellt die in der Probe gespeicherte elastische Energie dar. Aus Darstellungsgründen wurden $W_{\text {loss }}$ und $W_{\text {elast }}$ an unterschiedlichen Zyklen eingezeichnet. Das Verlustverhältnis $W_{\text {loss }} / W_{\text {elast }}$ muss sich natürlich auf die Messgrößen am gleichen Zyklus beziehen.

und als solcher eine Energiedichte. Er ist proportional zur Dichte der Bindungsenergie und bei konstanter Bindungsenergie eines Einzelatoms proportional zur Atomdichte. Im kristallinen als höher geordnetem $\mathrm{Zu}$ stand ist die Atomdichte größer als in der amorphen Phase. Damit ist bei einem Anstieg des Volumenanteils kristalliner Phase in PEO auch mit einem erhöhten EModul zu rechnen. Im Unterschied dazu fuihrt die räumliche Vernetzung der Kohlenwasserstoff-Ketten nicht zu einer Erhöhung der Atomdichte. Auch die mittlere Bindungsenergie pro Atom erhöht sich nur marginal, da die Mehrzahl der Bindungen innerhalb der Kette vorliegt. Die Dichte der Bindungsenergie steigt unwesentlich, und der E-Modul bleibt weitgehend unverändert. Durch die Ausbildung von Bindungen zwischen den Ketten wird aber die gegenseitige Kettenverschiebung erschwert, was die Härte erhöht und die Viskosität reduziert.

\section{Ausblick}

Die mechanische Charakterisierung von Nanostrukturen hat einen hohen Stellenwert und wird an Bedeutung weiter gewinnen. So muissen Halbleiterbauelemente auf ihre mechanische Stabilität geprüft werden. In zahlreichen Sensoren und Aktuatoren sind miniaturisierte Komponenten integriert, die mechanische Aufgaben erfuillen und entsprechend belastet werden (z. B. Flügelräder in Mikrodosierpumpen). Daraus erwächst ein hohes applikatives Potenzial der Nanohärteprüfung in der Industrie. Weiterhin verändert sich die Physik häufig in nichtlinearer, unerwarteter Weise, wenn man die Abmessungen der betrachteten Systeme nach unten skaliert. Damit ist die Nanohärtemessung für die Grundlagenforschung selbst dann interessant, wenn man kleine Eindrücke in großen Systemen ausfuihrt.

\section{Danksagung}

In die Arbeit sind Ergebnisse eingeflossen, welche der Autor B. Wolf an der TU Dresden (Institut fuir Kristallographie und Festkörperphysik) und an der Universität des Saarlandes gewonnen hat. Für die Unterstuitzung durch die betreffenden Einrichtungen bedanken sich die Autoren sehr herzlich. Ebenso gilt unser Dank der DFG, der Deutschen Akademie der Naturforscher "Leopoldina" Halle (Saale) sowie dem Fonds der Chemischen Industrie.

Ein weiterer Teil der Untersuchungen erfolgte im Rahmen des HSPN-Projektes „Qualitätssicherung von dünnen Filmen und Kompositwerkstoffen mittels Nanohärte“. Die Forschung an Polymeren wurde durch das IQN-Projekt „Technologie neuer Materialien“ sowie durch eine Zusammenarbeit mit dem Institut für Festkörperelektronik der TU Dresden möglich. Autor M. Nowicki dankt dem DAAD und dem ZIP-Programm für die finanzielle Unterstuitzung.

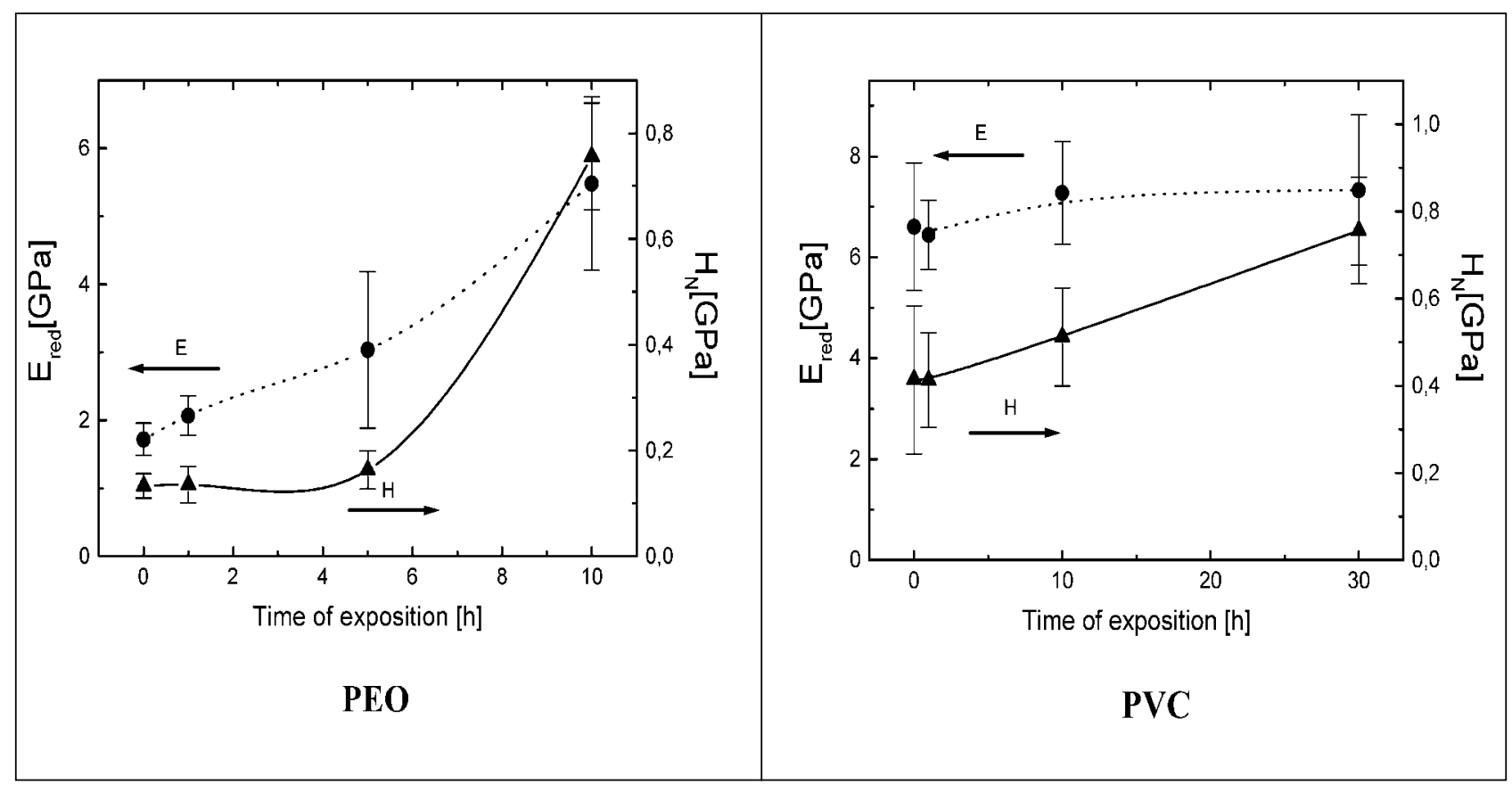

Abb. 11: Reduzierter E-Modul $E_{\text {red }}$ und Härte (mittlerer Kontaktdruck) $H_{N}$ als Funktion der Expositionsdosis (Bestrahlungsdauer) für die Polymere PEO (li.) und PVC (re.). 


\section{Literatur}

1 D. Tabor: The Hardness of Metals, Clarendon Press, Oxford, 1951

2 E. I. Tadmor, R. Miller, R. Phillips, M. Ortiz, J. Mater. Res. 14 (1999) 2233

3 B. Wolf, P. Paufler: Nanomechanical properties of brittle matter, in: NATO ASI proceedings „Fundamentals of tribology“, hg. von Bh. Bhushan, Serie II, Band 10, Kluwer, Dordrecht, 2001, 549-556

4 M. Kempf: Dissertation, Lehrstuhl Methodik der Werkstoffwissenschaften, Universität des Saarlandes, Saarbrücken, 2002

5 B. Bhushan, A. V. Kulkarni, W. Bonin, J. T. Wyrobek: Philos. Mag. A74 (1996) 1117

6 W. C. Oliver, G. M. Pharr, J. Mater. Res. 7 (1992) 1564

7 B. Wolf: Cryst. Res. Technol. 35 (2000) 377

8 J. McColm, Ceramic hardness, Kapitel 3.6 (theoretical models for hardness anisotropy), Plenum Press, New York, 1990, 97-116

9 M. Levy, H. E. Bass, R. R. Stern: Handbook of elastic properties of solids, liquids and gases, Academic Press, San Diego, 2001

10 J. J. Vlassak, W. D. Nix: Philos. Mag. A67 (1993) 1045

11 Yu. A. Osip'yan, V. F. Petrenko, A. V. Zaretskij, R. W. Whithworth: Advances in Physics 35 (1986) 115

12 B. Wolf, P. Paufler: phys. stat. sol. a 187 (2001) 415

13 M. Günther, B. Wolf: Fine Mechanics and Optics 46 (2001) 365

14 G. J. Ackland, Rep. Progr. Phys. 64 (2001) 483

15 H. Kaczmarek, Polymer Research 37 (1996) 547

16 H. Kaczmarek: Photochemische Effekte in Polymeren (in polnisch), UMK, Torun, 1988.

17 R. W. Cahn, P. Haasen, E. J. Kramer (Herausgeber): Material Science and Technology, Band 12: Structure and Properties of Polymers, VCH, Weinheim, 1993

18 J. Pielichowski, A. Pruszynski: Technologie der Polymere (in polnisch); WNT, Warszawa, 1994.

19 J. F. Rabek: Polymer Photodegradation - Mechanisms and Experimental Methods, Chapman \& Hall, London, 1993

\section{Autoren}

Dr. rer. nat. Bodo Wolf

Technische Fachhochschule Wildau

Labor für Oberflächentechnik

Tel. (0 33 75) 508-184

E-Mail: bwolf@igw.tfh-wildau.de

und

Technische Universität Dresden

Institut für Kristallographie und Festkörperphysik 01062 Dresden

Dipl.-Kristallograph Ronald Ries

Technische Fachhochschule Wildau Labor für Oberflächentechnik

Tel. (0 33 75) 508-217

E-Mail: rries@pt.tfh-wildau.de

Dr.-Ing. Marek Nowicki

Technische Universität Poznan

Institut für Physik

Ul. Nieszawska 13A, PL 60-965 Poznan, Polen

Tel. +48 (61) 665-31 96

E-Mail: mnowicki@phys.put.poznan.pl

Prof. Dr. rer. nat. Asta Richter, Vizepräsidentin

Technische Fachhochschule Wildau

Labor für Oberflächentechnik

Tel. (0 33 75) 508-219

E-Mail: richter@pt.tfh-wildau.de 